\title{
Satisfacción de los pacientes en tRatamientos por abuso de SUSTANCiAs Y SU RELACIÓN CON VARIABLES DE LAS INSTITUCIONES ASISTENCIALES*
}

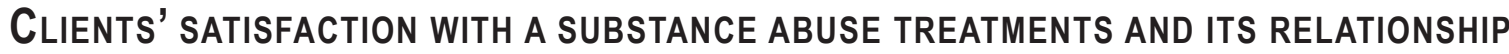 WITH THE INSTITUTION CHARACTERISTICS}

\author{
Andrés J. Roussos ${ }^{* *}$, Juan Martín Gómez Penedo*** y Julieta Olivera Ryberg****
}

\author{
*Estudio realizado a pedido de la Secretaría de Programación para la Prevención de la Drogadicción y la Lucha \\ contra el Narcotráfico de la República Argentina (SEDRONAR) y financiado por la Organización Internacional para \\ las Migraciones (OIM) \\ **Doctor en Psicología. Profesor Titular en la asignatura Metodología de la Investigación en Psicología en la Facultad \\ de Humanidades de la Universidad de Belgrano (UB), Profesor Adjunto en la asignatura Metodología de la \\ Investigación de la Facultad de Psicología de la Universidad de Buenos Aires (UBA), Director del Equipo de \\ Investigación en Psicología Clínica (EIPSI) en la Universidad de Belgrano (UB) y Miembro de la Carrera \\ del Investigador Científico del Consejo Nacional de Investigaciones Científicas y Técnicas (CONICET). \\ E-Mail: andres.roussos@comunidad.ub.edu.ar \\ Crisólogo Larralde 4921- Ciudad de Buenos Aires, Argentina. \\ ***Licenciado y Doctorando en Psicología. Becario Doctoral del Consejo Nacional de Investigaciones Científicas y \\ Técnicas (CONICET) y Miembro del Equipo de Investigación en Psicología Clínica (EIPSI) de la Universidad \\ de Belgrano (UB). E-Mail: juanmartin.gomez@comunidad.ub.edu.ar \\ ****Doctora en Psicología. Becaria Postdoctoral del Consejo Nacional de Investigaciones Científicas y Técnicas \\ (CONICET) y Miembro del equipo de investigación en Psicología Clínica de la Universidad de Belgrano (UB). \\ E-Mail: joliveraryberg@gmail.com \\ Universidad de Buenos Aires (UBA) \\ Consejo Nacional de Investigaciones Científicas y Técnicas (CONICET)
}

\section{RESUMEN}

La satisfacción que los pacientes tienen con respecto a sus tratamientos es un indicador de la calidad de los mismos y un importante predictor de sus posibles resultados. Se analizaron los niveles de tal satisfacción en una muestra de pacientes con problemáticas de abuso de sustancias, tratados en instituciones del Área Metropolitana de Buenos Aires (AMBA). Los grados de satisfacción fueron a su vez analizados en relación con las características diferenciales de las instituciones (tipo de institución, ubicación geográfica, género de los pacientes aceptados, presencia de cualidades atípicas en las instituciones) y la evolución de los tratamientos (duración y cantidad de interrupciones del proceso). A partir de un muestreo aleatorio por conglomerados y estratificado, se convocó una muestra de 216 participantes pertenecientes a 22 centros, que completaron un cuestionario de satisfacción, diseñado para este estudio. Los resultados mostraron niveles elevados de satisfacción en las distintas áreas exploradas, observando características distintivas de las instituciones asociadas a diferentes niveles de satisfacción, como es el caso del tipo de institución y el género de los participantes aceptados. No obstante, no se encontraron diferencias en los grados 
de satisfacción en función del tiempo de desarrollo del tratamiento y la cantidad de interrupciones del mismo. Los resultados obtenidos son discutidos en relación a los alcances del estudio, a la vez que se presentan sus limitaciones y se proponen futuras investigaciones para avanzar sobre esta temática.

Palabras clave: Satisfacción de los pacientes; Tratamiento; Abuso de sustancias; Variables de las instituciones; Área Metropolitana de Buenos Aires.

\section{ABSTRACT}

The client satisfaction about his treatments it is an important indicator of its quality, but also a fundamental predictor of treatment outcome. Specifically, in patients with drug abuse disorders, the degrees of satisfaction with their treatments have presented lineal correlations with the amount of therapeutic change and the continuity in treatment (prevention of drop-out). Thus, the analysis of clients 'satisfaction with their treatments is presented as an indirect and alternative strategy to analyze the effects of therapy for drug abuse disorders, a main health, social, and political problem in the world wide. In this exploratory study we analyzed the levels of satisfaction about their treatment in a sample of client's with substance abuse disorders, treated in institutions of the Buenos Aires Metropolitan Area (AMBA). Then we explored the relationship among the levels of satisfaction with the specific features of the institutions where the treatments were conducted (type of institution, geographic location, genre of the clients accepted, and the presence of atypical characteristics) and with characteristics of the evolution of those treatments (treatments length and amount of interrup- tions during the therapeutic process). Based on a stratified cluster random sampling strategy, using the above-described characteristics of the institutions analyzed in this study to create the clusters, we designed a sample of 216 subjects that were treated in 22 institutions with treatments subsidized by the Argentine National Secretary for the Prevention of Drug Abuse and the Fight against Drug Trafficking (SEDRONAR by its acronym in Spanish). All the participants of this research completed a question- naire developed ad hoc and based on previous developments, to explore patient's levels of satisfaction in six areas: Admission, General environment (divided into the sub-areas Human and physical context and Mistreatment), Satisfaction with food, Staff evaluation (divided into the sub-areas Overall staff assessment and Specific staff assessment), Satisfaction with treatment (divided into the subareas Treatment features, Treatments results, and Complementary therapeutic devices), and General treatment evaluation. Results showed that there were same features of the institutions related to different levels of satisfaction in the participants of the study. Regarding the type of institution, patients of the psychiatric clinics presented the lowest degrees of satisfaction while the patients of the day hospitals had the highest levels of satisfaction. Also different levels of satisfactions were observed based on the genre of the patients accepted in the institutions. Patients of the institutions that only accept male clients have greater degrees of satisfaction than patients in mixed institutions. No significant differences were found in patient's satisfaction due to institution geographical location. Also, we have not found a significant association between patient's satisfaction and treatment length or between treatment satisfaction and amount of interruptions in the therapy.

The results are discussed in the context of the scopes of the study, while further research is suggested to move forward in the exploration of this issue. The differences in satisfaction due to the type of institution might be explained by the degree of openness and flexibility of the setting, but also by the severity of the patients that are included in each type of institutions. As well, the differences in satisfaction based on the genre of the patients accepted by the institution could be explained by the predominance of male patients in the population $(85 \%)$ and the possible lack of adaptation of treatment characteristics to female specific necessities. However, the results of this research must be interpreted extremely cautious because of limitations of the study related to the sample method and the unknown psychometric properties (in terms of validity and reliability) of the instrument used.

Key words: Clients' satisfaction; Treatment; Drug abuse; Institutional characteristics; Buenos Aires Metropolitan Area. 


\section{INTRODUCCIÓN}

Los trastornos por abuso y dependencia de sustancias tanto lícitas como ilícitas, representan un problema social y político a nivel global según datos de la Oficina de las Naciones Unidas contra las Drogas y el Delito (ONUDD, 2003), asociado a diversas consecuencias perjudiciales tales como exclusión social, delincuencia, trastornos familiares, propagación de enfermedades infecciosas, uso excesivo de los servicios de salud y pérdida de productividad, entre otras (ONUDD, 2012; OPS, 2009). Los abordajes terapéuticos para esta problemática junto con las políticas de prevención juegan un rol primordial en la reducción de estos riesgos y consecuencias perjudiciales asociadas al consumo abusivo, favoreciendo la disminución de su impacto negativo sobre la sociedad y sus individuos.

A nivel mundial, los tratamientos existentes han mostrado su utilidad para el abordaje de esta problemática, tanto a partir de estudios clínicos controlados como de investigaciones en contextos naturales (ONUDD, 2003).

No obstante, la Organización Mundial de la Salud (OMS, 2003) insta a organismos públicos y privados a mantener un constante seguimiento de la calidad de estos tratamientos como así también recomienda utilizar estrategias alternativas a las recién mencionadas para su evaluación. Entre otras estrategias, la OMS (2003) sugiere el estudio de la satisfacción de los pacientes con los tratamientos, como un indicador importante de su calidad, al igual que la evaluación de los cambios en los síntomas y la funcionalidad de los pacientes. Si bien históricamente la evaluación de los tratamientos y sus efectos se han centrado en los estudios de eficacia, efectividad y eficiencia (Lambert \& Ogles, 2004), los estudios de satisfacción han proliferado en los últimos años como una alternativa para evaluar la calidad de los abordajes. El nivel de satisfacción de un paciente es ante todo una medida de orden subjetivo. Su función es dar cuenta del grado de similitud percibida por el paciente entre el tratamiento recibido y la representación que tiene del abordaje ideal (Arenas, Fuentes \& Campos, 1993). Los es- tudios de satisfacción han generado un interés creciente en los investigadores clínicos en los últimos años, ya que permiten estudiar la perspectiva particular que los pacientes tienen y los juicios que realizan con respecto a sus tratamientos (Holcomb, Parker, Leong, Thiele \& Higdon, 1998).

Por otra parte, además de ser un indicador de calidad en sí mismo, la satisfacción se ha visto asociada a los niveles de mejoría en los tratamientos, aumentando en consecuencia el interés por su estudio. Por ejemplo, autores como Ankuta y Abeles (1993) y Pekarik y Wolff (1996) han reportado correlaciones directas significativas entre los niveles de satisfacción y la presencia de cambios terapéuticos positivos, medidos tanto desde la perspectiva del paciente como de la del terapeuta.

En el caso particular de pacientes con trastornos por abuso de sustancias, se ha observado que la satisfacción tiene una relación directa con los resultados del abordaje y el mantenimiento de los pacientes en los tratamientos. Mayores niveles de satisfacción se han visto asociados a menor cantidad de consumo de sustancias durante el abordaje (Kelly, O’Grady, Brown, Mitchell \& Schwartz, 2010; Morris \& Gannon, 2008), al finalizar el tratamiento (Marchand, Oviedo-Joekes, Guh, Brissette, Marsh \& Schechter, 2011) y en seguimientos posteriores (Zhang, Gerstein \& Friedmann, 2008). Por otra parte, mayores niveles de satisfacción se han relacionado con menor cantidad de abandonos de los tratamientos (Kelly et al., 2010; Marchand et al., 2011). A su vez, algunos autores consideran que la satisfacción de los pacientes puede estar vinculada a componentes cruciales del proceso terapéutico como es el caso de la alianza terapéutica (Bunge, 2012).

En términos generales, el estado del arte ha mostrado altos niveles de satisfacción en diversas propuestas terapéuticas para pacientes con abuso o dependencia de sustancias. Se han reportado elevados puntajes en escalas de satisfacción con respecto a programas generales de tratamiento para adiciones (Cernovsky, O'Reilly \& Pennington, 1997; Mc Lellan \& Hunkeler, 1998), tratamientos con metadona (Kelly et al., 2010), 
con farmacoterapia (Frick et al., 2011; Zhang et al., 2008), psicoterapéuticos (Donovan, Kadden, Diclemente \& Carroll, 2002), residenciales / de internación y tratamientos comunitarios (Morris \& Gannon, 2008). Programas generales de abordaje para pacientes duales mostraron también altos niveles de satisfacción (Schulte, Meier \& Stirling, 2011).

En la exploración del estado del arte realizada para este estudio se han encontrado escasas investigaciones conducidas en Latinoamérica sobre satisfacción de tratamientos en pacientes con abuso de sustancias. En Perú, Rojas Valero y Espinoza Paul (2008) evaluaron los niveles de satisfacción con tratamientos basados en intervenciones cognitivo-conductuales y humanísticas, observando altos niveles de satisfacción. El 46\% de los pacientes manifestó que el tratamiento era excelente, mientras que el $50 \%$ percibió que era bueno. Solo el 3.1\% sostuvo que la calidad del tratamiento era regular.

Por otro lado, el Consejo Nacional para el Control de Estupefacientes (s.f.) de Chile, estudió los niveles de satisfacción de pacientes con abuso de sustancias, comparando un plan básico de tratamiento con un tratamiento intensivo y otro residencial. El plan básico presentó los niveles de satisfacción más altos en cuanto a las actividades realizadas, el compromiso con el programa, el trato del personal, las relaciones humanas establecidas, el grado de cumplimiento de las expectativas previas y la reinserción social posterior al tratamiento. En cambio, el programa intensivo mostró mayores niveles de satisfacción con respecto a las capacidades técnicas de los profesionales, la infraestructura de las instituciones y el acceso a los centros. El programa residencial tuvo el mismo nivel de satisfacción que el intensivo con respecto a la capacidad técnica de los profesionales, pero en el resto de las áreas presentó los niveles más bajos de satisfacción.

En la búsqueda bibliográfica para al desarrollo de este proyecto de investigación, no se encontraron estudios realizados en la República Argentina sobre satisfacción con los tratamientos en pacientes con esta problemática.
No obstante, algunos resultados preliminares del proyecto de investigación más amplio, en el que se enmarca este estudio, fueron publicados por Gómez Penedo, Barreiro y Roussos (2015). En dicho trabajo los autores exploraron la asociación entre características previas de los pacientes (demográficas y clínicas) con sus niveles de satisfacción en su tratamiento por el abuso de sustancias. Se observaron niveles significativamente mayores en el género masculino y en pacientes con menores grados de instrucción académica. A su vez, pacientes con menor grado de complejidad en sus problemáticas clínicas (menor cantidad de sustancias consumidas que motivaron la consulta) y con mayores redes sociales de apoyo (por ejemplo, aquellos que estaban en pareja), reportaron niveles de satisfacción más altos por el tratamiento. En base a estos resultados los autores llaman a valorar estas características previas de los pacientes, al momento de diseñar los tratamientos para pacientes con problemáticas por abuso de sustancias.

En continuidad con esos análisis, se deduce que también puede ser relevante explorar cómo las características de las instituciones donde se desarrollan los tratamientos se asocian con los niveles de satisfacción de sus pacientes. En este contexto, el objetivo general de esta investigación fue analizar los niveles de satisfacción en pacientes tratados por consumo abusivo de sustancias en instituciones del Área Metropolitana de Buenos Aires (AMBA), indagando la relación de dichos niveles con variables diferenciales de las instituciones y la evolución de los tratamientos.

De esta forma, el objetivo específico de la investigación realizada fue estudiar los niveles de satisfacción de los pacientes y su relación con: (1) el tipo de instituciones donde realizaron los tratamientos, (2) su ubicación geográfica, (3) el género de los pacientes aceptados en las instituciones, (4) la existencia de características atípicas de las instituciones y (5) el tiempo de desarrollo del tratamiento y sus interrupciones.

Se considera que un estudio de esta naturaleza puede aportar datos para el establecimiento de políticas públicas, brindando un 
panorama general sobre la opinión de los pacientes drogodependientes acerca de sus tratamientos.

Investigaciones en esta dirección podrían representar un recurso importante para promover la salud mental, ya que implican un aporte para la comprensión de los fenómenos que en ella impactan (Bunge, 2012). Del mismo modo, este estudio intenta sentar las bases para futuros estudios de efectividad y eficacia en estas poblaciones, orientados a investigar los efectos de las distintas propuestas terapéuticas en el abordaje de estos pacientes.

\section{MÉTodo}

\section{CARACTERIZACIÓN DE LOS TRATAMIENTOS}

La Secretaría de Programación para la Prevención de la Drogadicción y la Lucha contra el Narcotráfico de la República Argentina (SEDRONAR) cuenta con un sistema de evaluación y derivación para pacientes que se acercan voluntariamente o que son derivados a ella por vía judicial, para realizar un tratamiento por abuso de sustancias. Un equipo interdisciplinario de profesionales evalúa al paciente y la severidad de la problemática. Luego, el equipo selecciona la institución adecuada para el tratamiento, en base a una clasificación de los centros en cuatro categorías (A, B, C y D), según las modalidades de tratamiento que ofrecen y el grado de complejidad de los pacientes (Observatorio Argentino de Drogas -OAD-, 2009).

Las instituciones Tipo A son clínicas psiquiátricas a las que se derivan pacientes duales (trastornos psiquiátricos comórbidos con la adicción) y que trabajan exclusivamente con tratamientos de internación.

Las instituciones Tipo B son comunidades terapéuticas con guardia psiquiátrica y control psiquiátrico, en ellas se atiende tanto a pacientes duales como no duales mediante abordajes ambulatorios o de internación.

Las Tipo $\mathrm{C}$ son comunidades terapéuticas sin guardia psiquiátrica que incluyen tratamientos ambulatorios o con internación.
Las instituciones Tipo D son hospitales de día y trabajan exclusivamente con tratamientos ambulatorios.

En todas las instituciones se ofrece terapia individual y grupal, junto con diversos talleres y actividades de esparcimiento. Solo en las instituciones de tipos A y B, los tratamientos pueden ser combinados con farmacoterapia.

A los pacientes incluidos en el programa se les financia completamente el tratamiento por un año, teniendo la posibilidad de renovar el subsidio por otro año más, de ser necesario.

Más allá de la clasificación planteada, resulta difícil realizar una caracterización unívoca de los tratamientos aplicados en las instituciones subsidiadas por la SEDRONAR, debido a su alto grado de heterogeneidad, tanto desde el punto de vista del marco teórico como de las técnicas terapéuticas utilizadas y el tipo de dispositivos que brindan, a saber: talleres, actividades recreativas, cursos de capacitación, etc. Cada institución presenta su propio programa de tratamiento integrando intervenciones y nociones de diversos marcos teóricos al momento de conceptualizar a los pacientes y planificar su abordaje.

En busca de un criterio unificador para los tratamientos para toxicomanías, la ONUDD (2003) los define como abordajes integrados por una o más intervenciones, con cierto grado de estructuración, orientados a mejorar los problemas de salud de los pacientes y a restablecer un desarrollo a nivel social y personal adecuado. A pesar de su heterogeneidad, todos los tratamientos de las instituciones subsidiadas cumplen con la definición planteada por la ONUDD, incluyéndose así en el marco conceptual de lo que sería considerado un tratamiento para toxicomanías según estándares internacionales. Además, como sostiene el Observatorio Argentino de Drogas (2009), a pesar de las diferencias en las modalidades de tratamiento, todas las instituciones subsidiadas por la SEDRONAR coinciden en una serie de objetivos comunes: logro de abstinencia, reestructuración del entorno social y familiar, adquisición de habilidades o técnicas de afrontamiento, reinserción en la sociedad y establecimiento de un proyecto de vida. 


\section{PARTICIPANTES}

La muestra del estudio estuvo conformada por pacientes que se encontraban realizando algún tratamiento por abuso o dependencia de sustancias subsidiado por la SEDRONAR, en alguna de sus instituciones en el Área Metropolitana de Buenos Aires (AMBA).

Para el diseño de la muestra se utilizó un muestreo aleatorio por conglomerados y estratos. El marco muestral estuvo integrado por todas las instituciones dedicadas al tratamiento de pacientes con trastornos por abuso o dependencia de sustancias ubicadas en el AMBA que contasen con un mínimo de tres pacientes subsidiados por la SEDRONAR, siendo cada institución un conglomerado de la muestra. Dichos conglomerados fueron divididos en estratos en función de las variables de interés presentadas en los objetivos específicos: tipo de institución, género de los pacientes admitidos (instituciones mixtas o instituciones que solo aceptan pacientes varones) y ubicación geográfica.

Se seleccionaron al azar 22 centros con un total de 279 potenciales participantes. Posteriormente, dos instituciones debieron ser reemplazadas por otras, por no contar con pacientes subsidiados al momento de realizar el trabajo de campo.

A continuación se describe la distribución de instituciones seleccionadas para la muestra según sus características. Entre paréntesis se aclara la cantidad de instituciones con dichas características en la población objetivo:

- Ubicación: Se seleccionaron 5 instituciones que se encontraban en la Ciudad Autónoma de Buenos Aires [CABA] (de 9 que integraban la población), 5 ubicadas al norte de la CABA (de 12), 9 al sur (de 15) y 3 al oeste (de 7).

- Tipo de institución: La única institución de Tipo A presente en la población fue incluida en la muestra. Además, se estudiaron 5 de Tipo B (de un total de 10), 12 de Tipo C (de un total de 25) y 4 de Tipo D (de un total de 7).

- Género de los pacientes aceptados: 9 instituciones estudiadas era mixtas (de 17 presentes en la población) y 13 solo aceptaban hombres (de 26).
Del total de 279 pacientes incluidos en los 22 centros seleccionados, 216 sujetos contestaron el cuestionario de satisfacción. Esto se debe a pacientes que no se encontraban en la institución al momento de la administración del cuestionario y sujetos que finalizaron el tratamiento en el lapso entre el diseño de la muestra y la administración del cuestionario.

El promedio de edad de los participantes fue igual a 31 años $(D E=9.16)$. El promedio de edad en las mujeres fue igual a 36 años $(D E=11.15)$, mientras que en los varones fue igual a 30.20 años $(D E=8.5)$. El $84.8 \%$ de los sujetos pertenecía al género masculino. El $78 \%$ de los participantes era soltero, el $4.3 \%$ se encontraba separado, el $5.7 \%$ divorciado, el . $5 \%$ viudo, el $8.6 \%$ estaba casado y el $2.9 \%$ en pareja.

Con respecto a los niveles de escolaridad, el $14.3 \%$ tenía estudios primarios incompletos, el $17.6 \%$ primarios completos, el $45.7 \%$ secundarios incompletos, el $13.3 \%$ secundarios completos, el $6.2 \%$ terciarios incompletos y el $2.9 \%$ terciarios completos.

El 25.4\% de la muestra llevaba menos de un mes en tratamiento, el $51.7 \%$ menos de 3 meses y el $83.1 \%$ menos de 6 meses.

En el Gráfico 1 se presenta la distribución de los participantes según la cantidad de meses desde el inicio del tratamiento. La distribución asimétrica positiva del tiempo en tratamiento se puede explicar por las altas tasas de abandono observadas en este tipo de tratamientos, que según datos de un estudio realizado previamente en el AMBA es del $66.7 \%$, teniendo los pacientes de estos abordajes una media de permanencia en los tratamientos de 121 días (OAD, 2009).

\section{INSTRUMENTOS}

La variable Satisfacción de los pacientes por sus tratamientos fue definida operacionalmente mediante un cuestionario anónimo de 49 ítems de tipo escala Likert diseñado ad hoc en base a la Patient Satisfaction Survey, desarrollada por la New York State Office of Alcoholism and Substance Abuse Service Bureau of Addiction Treatment Centers (s.f.). 
Se diseñó este cuestionario especialmente para este estudio y los autores analizaron las características de la encuesta recién citada. Los ítems en inglés en su formato original, considerados pertinentes, fueron traducidos y luego incorporados al cuestionario, mediante un procedimiento de back-translation. A esos fines, el segundo autor del estudio tradujo los ítems al español y el primero los tradujo nuevamente al inglés para observar si se encontraban diferencias entre ellos. Al no encontrarse diferencias sustanciales, se incorporó la traducción de los ítems. Por otra parte, se modificaron algunos ítems de la escala original, adaptándolos a los objetivos de este estudio y otros se agregaron, en función de las áreas específicas de interés de esta investigación.

Las respuestas se ubicaron en una escala de 1 (Muy insatisfecho / Poco) a 5 (Muy satisfecho / Mucho) y los ítems se distribuyeron en seis clusters (divididos en algunos casos en subclusters) que representan distintas áreas de satisfacción asociadas al abordaje proporcionado por la institución. Se describen las definiciones operacionales de los distintos clusters y subclusters, mediante los ítems utilizados:

\section{1.- Cluster de AdMISIÓN E INGRESO}

En este cluster se incluyeron ítems que indagaban la forma en que fue tratado el paciente durante el proceso de admisión y la facilidad para acceder al servicio. A su vez, examinaban el proceso de presentación de las instalaciones, los profesionales y los demás pacientes durante la inserción al tratamiento.

\section{2.- Cluster de Ambiente general (Dividido en} LOS SUBCLUSTERS ÁMBITO FÍSICO Y HUMANO Y MALTRATO)

En la Escala de Ambiente General se exploró el nivel de satisfacción de los pacientes con respecto al entorno y al medio en el que se llevó a cabo el programa de tratamiento, como así también aspectos no específicos de los tratamientos, pero que formaron parte del dispositivo en el que el mismo se desarrolló. - SUBCLUSTER DE AMBIENTE FÍSICO Y HUMANO

Con esta subescala se evaluó la satisfacción de elementos extra-terapéuticos, tales como las condiciones edilicias o las relaciones del paciente con sus compañeros.

- Subcluster de MALTRATO

Con esta subescala se evaluó la presencia de violencia física, agravios o humillaciones en actividades terapéuticas o extra-terapéuticas, por parte de terapeutas, empleados de la institución o compañeros del paciente.

\section{3.- Cluster de Comida}

Con esta subescala se exploró la satisfacción de los pacientes con la cantidad y calidad de comida suministrada por la institución.

4.- Cluster de Evaluación del personal (DIVIDIDO EN LOS SUBCLUSTERS EVALUACIÓN GENERAL DEL PERSONAL Y EVALUACIÓN ESPECÍFICA DEL PERSONAL)

Con este cluster se evaluó la satisfacción de los pacientes con respecto al personal de la institución. Este cluster estuvo divido, a su vez, en dos conjuntos:

- SUbCLUSTER DE EVALUACIÓN GENERAL DEL PERSONAL

Con este subcluster se indagó la satisfacción en forma global con el personal a cargo de la asistencia del paciente.

- SUBCLUSTER DE EVALUACIÓN ESPECÍFICA DEL PERSONAL

Los ítems de este subcluster evaluaron la satisfacción específica con respecto a los diferentes empleados y profesionales de la institución, a saber: operadores terapéuticos, psicólogos, psiquiatras, médicos generales, asistentes sociales, enfermeros y directores.

\section{5.- Cluster de Tratamiento}

Con esta subescala se analizó la satisfacción con respecto a los diversos componentes que integraron el tratamiento. Este apartado se dividió en tres subclusters:

- SUBCLUSTER DE CARACTERÍSTICAS DEL TRATAMIENTO

Sus ítems exploraron la satisfacción con las propuestas terapéuticas de la institución (psicoterapia, tratamiento farmacológico, terapia de grupos, etc.).

- SubCluster de RESUltados DEL tRATAMIENTO

Con esta subescala se observó el grado de satisfacción con los resultados obtenidos a partir del tratamiento. 


\section{- SUBCLUSTER DE DISPOSITIVOS COMPLEMENTARIOS}

Con este subcluster se evaluó la satisfacción con actividades extra-terapéuticas incluidas en el programa general de tratamiento (talleres, actividades deportivas, actividades educativas, etc.).

\section{6.- Cluster de Evaluación general del trata- MIENTO}

Con sus ítems se examinó la satisfacción global de los pacientes en relación con el tratamiento brindado por la institución.

Debido a que el cuestionario de satisfacción original, en el que se basa el instrumento utilizado en este estudio, no fue diseñado con fines de investigación, sino para una evaluación y seguimiento de la satisfacción de pacientes por abuso de sustancias en el estado de Nueva York, no se han explorado sus propiedades psicométricas en términos de confiabilidad y validez. Del mismo modo, al ser una traducción ad hoc realizada a los fines de este estudio, no se ha realizado una adaptación al contexto argentino del instrumento usado en esta investigación, ni se han evaluado previamente sus niveles de confiabilidad y validez.

Sin embargo, para tener una aproximación de la confiabilidad de la escala se presentan a continuación los coeficientes Alpha de Cronbach (como evidencia de consistencia interna) y el promedio de las correlaciones ítem-escala ajustadas (como evidencia de la homogenedidad de los ítems), del cuestionario total, los clusters y los subclusters (ver Tabla 1).

No se realizaron análisis de la validez del instrumento, debido a la falta de información empírica pertinente a estos fines.

Al finalizar el cuestionario, los pacientes debían responder preguntas acerca de sus datos demográficos y también dos preguntas adicionales del tratamiento que se utilizaron para operacionalizar variables de interés en este estudio: cuánto tiempo llevaba el paciente en tratamiento y cuántas interrupciones había tenido.

La SEDRONAR aportó información acerca de características de las instituciones tales como su ubicación geográfica, tipología, gé- nero de los pacientes que admiten y la presencia de cualidades atípicas (ser instituciones religiosas o instituciones suspendidas en algún momento por la SEDRONAR). Esos datos se usaron para la creación de los estratos aplicados en el diseño muestral y a su vez, conformaron la operacionalización de la variable características de las instituciones, para los posteriores análisis realizados en este estudio.

\section{Procedimientos}

Las dos instituciones que reemplazaron a los centros excluidos de la muestra por falta de sujetos, fueron seleccionadas al azar de los estratos a los que pertenecían las que debieron ser sustituidas.

La administración de los cuestionarios fue realizada en las 22 instituciones por dos colaboradores de la SEDRONAR, quienes coordinaron días y horarios con las instituciones para su aplicación.

Los colaboradores debían entregar los cuestionarios a todos los pacientes pertenecientes y leer sistemáticamente cada ítem. Conforme se leían las preguntas, los pacientes debían contestarlas individualmente en su cuestionario personal.

La recolección de los datos comenzó en noviembre del año 2012 y culminó en enero del año 2013.

\section{ANÁLISIS ESTADÍSTICOS}

Se aplicó la prueba de normalidad Kolmogorov-Smirnov a los resultados obtenidos tanto de los ítems del cuestionario como de los distintos clusters y subclusters, para determinar si su distribución se ajustaba a una distribución normal. En todos los casos, el estadístico arrojó valores de probabilidad por debajo del puntaje crítico de significación de .05 , rechazándose de esta manera la hipótesis nula de que la distribución de los ítems se ajustaba a una distribución normal.

La observación de los gráficos de distribución de los ítems fue consistente con esta 
conclusión. Por ello se utilizaron modelos estadísticos no paramétricos para el análisis de los datos. Para la comparación de dos muestras independientes se utilizó el estadístico $U$ de Mann-Whitney, mientras que para la comparación de más de dos muestras independientes se usó el $H$ de Kruskal-Wallis.

\section{Resultados}

En las Tablas 2 y 3 se presentan las medias de satisfacción de los participantes, para los clusters y subclusters de intéres, respectivamente, tanto en la muestra total como en función de las distintas características de las instituciones.

\section{SATISFACCIÓN GENERAL}

Como se puede observar en las Tablas 2 y 3 , con excepción del cluster Comida $(M=3.74)$ y el subcluster Dispositivos complementarios $(M=3.92)$, todos presentan un alto nivel de satisfacción por encima de una media igual a 4, que representa un alto grado de satisfacción.

\section{SATISFACCIÓN SEgÚN TIPO DE INSTITUCIÓN}

Como se presenta en las tablas de Resultados, las medias de satisfacción obtenidas de las instituciones A (Clínica psiquiátrica) son menores a las de las otras clases de instituciones, en los seis clusters estudiados. Por otra parte, las medias de las instituciones Tipo D (Hospital de día) fueron mayores a los de los otros tipos de instituciones en cinco de los seis clusters (con excepción del cluster Tratamiento). Sin embargo, debido al escaso número muestral de las instituciones $\mathrm{A}(n=9)$ y $\mathrm{D}(n=17)$, no pudieron realizarse comparaciones estadísticas entre ellas y las instituciones B y C. No obstante, mediante un análisis $U$ de Mann-Whitney para dos muestras independientes, se observó que aquellos institutos con guardia psiquiátrica (Tipo B) presentan grados de satisfacción significativamente menores que los institutos sin guardia psiquiátrica (Tipo C) en el cluster de Tratamiento $(M$ de instituciones B: $4.10 ; M$ de instituciones C: 4.24; $U$ de Mann-Whitney: 367113; $p \leq .05)$ y en los subclusters de Características del tratamiento ( $M$ de B: 4.04 ; $M$ de C: 4.23; $U$ de Mann-Whitney: 395467; $p \leq .05)$ y Resultados ( $M$ de B: $4.40 ; M$ de C: 4.47; $U$ de Mann-Whitney: 93306; $p \leq .05$ ) (ver Tabla 3).

\section{SATISFACCIÓN CON LA UBICACIÓN GEOGRÁFICA DE LAS INSTITUCIONES}

Se estudió esta característica, considerando que la ubicación de las instituciones podía relacionarse con diversos niveles socioeconómicos de los pacientes y que estos podían afectar los niveles de satisfacción.

La localización geográfica se estableció en base a su ubicación con respecto al AMBA y fueron clasificadas según se encontraban al norte, sur u oeste del AMBA, o en referencia específica a la Ciudad Autónoma de Buenos Aires (CABA).

Para establecer potenciales diferencias significativas originadas en la región se corrió una prueba no paramétrica para muestras independientes $H$ de Kruskal-Wallis. Se observó solo en el clusters de Comida una diferencia significativa $\left(\chi^{2}=7.894 ; p \leq .05\right)$ en favor de las instituciones ubicadas en la zona oeste, tanto en la comparación de medianas como en la comparación de las distribuciones. Sin embargo, dicho resultado no se vio asociado a hipótesis alguna relevante al respecto.

\section{SATISFACCIÓN POR GÉNERO DE LOS PACIENTES AD- MITIDOS EN LA INSTITUCIÓN}

En Resultados, las tablas informan las medias de satisfacción en función del género de los pacientes atendidos en las instituciones (Instituciones mixtas / Solo varones).

A partir del estadístico $U$ de Mann-Whitney se estableció que los pacientes de instituciones donde solo se atendían varones, mostraron niveles significativamente mayores de satisfacción en los clusters de Evaluación del 
personal $(M$ de Instituciones mixtas: 4.18; $M$ de instituciones donde solo se tratan varones: 4.26; $U$ de Mann-Whitney: 581559.5; $p \leq .05$ ) y Tratamiento ( $M$ de I. mixtas: 4.10 ; $M$ de Solo varones: 4.21; $U$ de Mann-Whitney: 1829407; $p \leq .05$ ), y en particular en los subclusters de Evaluación general del personal ( $M$ de I. mixtas: 4.25; $M$ de Solo varones: 4.37; $U$ de Mann-Whitney: 77135; $p \leq .05)$, Características del tratamiento $(M$ de I. mixtas: 4.03; $M$ de Solo varones: 4.19; $U$ de Mann-Whitney: 539826; $p \leq .05)$ y Dispositivos complementarios ( $M$ de I. mixtas: 3.86 ; $M$ de Solo varones: 3.95; $U$ de Mann-Whitney: $67180 ; p \leq .05)$.

\section{SATISFACCIÓN EN RELACIÓN CON LAS CARACTERÍS- TICAS ATÍPICAS DE LAS INSTITUCIONES}

En las tablas de la sección Resultados también se explicitan las medias de satisfacción de los pacientes en función de la presencia/ ausencia de dos características atípicas en sus instituciones: si eran religiosas o si en algún momento fueron temporalmente suspendidas por la SEDRONAR por alguna irregularidad.

El escaso número de pacientes en instituciones religiosas $(n=15)$ e instituciones suspendidas $(n=10)$, impidió estudiar la existencia de diferencias significativas desde el punto de vista estadístico en la satisfacción, en relación con estas características atípicas.

SATISFACCIÓN EN RELACIÓN CON EL TIEMPO DE DESARROLLO DEL TRATAMIENTO E INTERRUPCIONES DEL MISMO

Las medias de satisfacción en base a la presencia / ausencia de interrupciones del tratamiento también fueron evaluadas (ver Tablas 2 y 3 ). La prueba $U$ de Mann-Whitney no arrojó diferencias significativas en los niveles de satisfacción con respecto a la presencia o ausencia de ambas en el tratamiento. Asimismo, mediante el estadístico $H$ de Kruskal-Wallis no se encontró relación alguna entre la cantidad de meses en tratamiento y la satisfacción. Es decir, no se pudo hallar algún tipo de relación estadística que asocie la cantidad de tiempo que tienen los pacientes en tratamiento, ya sea poco o mucho, con un nivel de satisfacción diferencial.

\section{Conclusiones}

Tal como se puede observar en Resultados, el grado de satisfacción general de los pacientes fue alto, con los tratamientos y con los aspectos ambientales relacionados con dichos tratamientos. Estos altos niveles de satisfacción se observaron, en términos generales, tanto en los seis clusters como en los siete subclusters delimitados. El instrumento utilizado, como se menciona en la sección Métodos, contó con una escala de respuesta para sus ítems de 1 (Muy insatisfecho) a 5 (Muy satisfecho), observándose únicamente un cluster (Comida) y un subcluster (Dispositivos complementarios) con medias de satisfacción ligeramente inferiores a $4(M=3.37$ y $M=3.92$, respectivamente). Dicho resultado cobra mayor relevancia en función de los estudios que vinculan altos niveles de satisfacción con mejores resultados, en los tratamientos de pacientes con trastornos por abuso de sustancias (Kelly et al., 2010; Marchand et al., 2011; Morris \& Gannon, 2008; Zhang et al., 2008). Sin embargo, en este punto es necesario remarcar que la satisfacción de los tratamientos no es una medida equivalente a su eficacia o efectividad. Si se quisiera estudiar la calidad de los tratamientos, mediante el análisis de sus efectos en distintas áreas asociadas a la salud y problemática de los pacientes, serían necesarios otros dispositivos metodológicos.

A pesar de que en general la muestra contó con una media alta de satisfacción, se observa que dichos niveles no fueron homogéneos en las distintas instituciones, pudiendo identificarse algunas características específicas de los centros de tratamiento asociadas a mayores o menores grados de satisfacción.

Tal es el caso de las diferencias reportadas en función del tipo de institución en donde se realizaba el tratamiento. Diversas hipótesis se pueden plantear en relación con estas diferen- 
cias por tipo de institución. Por un lado se podría pensar que los niveles de satisfacción se ven asociados al grado de apertura y libertad en los tratamientos. Las instituciones psiquiátricas (Instituciones A), que cuentan con tratamientos de internación con un alto grado de control sobre los pacientes, mostraron los niveles de satisfacción más bajos. En contrapartida, los hospitales de día (Instituciones D) que ofrecen tratamientos ambulatorios con un alto grado de flexibilidad y libertad a los pacientes, presentaron los mayores niveles de satisfacción. Del mismo modo, al comparar comunidades terapéuticas con y sin guardia psiquiátrica, las que carecían de guardia psiquiátrica presentaron mayores niveles de satisfacción. En este sentido, se podría plantear que el hecho de que los tratamientos sean ambulatorios o de internación, tiene una repercusión importante en la satisfacción de los pacientes, siendo los tratamientos ambulatorios, que presentan una mayor apertura y libertad, los que informan medias de satisfacción más altas.

Sin embargo, puede contemplarse una hipótesis alternativa. A pesar de no contar con datos diagnósticos de los pacientes, se asume que las instituciones psiquiátricas y las comunidades terapéuticas con guardia psiquiátrica albergan una mayor cantidad de pacientes duales (comorbilidad entre consumo de sustancias y otro trastorno psiquiátrico) y, por ende, pacientes con un grado de severidad mayor. De esta forma, la complejidad de la problemática de los pacientes podría representar una variable moderadora crucial del grado de satisfacción y, a su vez, del pronóstico del paciente y su probabilidad de mejoría.

En ese caso, debería brindarse una mayor atención al abordaje de estos pacientes más complejos, tanto desde el punto de vista del tratamiento como de las condiciones ambientales en las que se enmarca, para optimizar su satisfacción, mejorando la probabilidad de alcanzar buenos resultados.

No obstante, el estudio realizado al no poseer datos sobre las características diagnósticas de los pacientes, no cuenta con la información primordial para el testeo de estas hipótesis. Futuras investigaciones deberían centrarse en explorar el rol y la interacción de estas variables sobre la satisfacción y, a su vez, la efectividad de estos tratamientos.

Otra variable asociada a diferentes niveles de satisfacción fue el género de los pacientes admitidos en las instituciones. Los pacientes de instituciones donde solo se atendían varones, mostraron niveles significativamente mayores de satisfacción en las escalas de Evaluación del personal (Subescala de Evaluación general del personal) y Tratamiento (Subescala de Características del tratamiento y dispositivos complementarios). Al no haber en la población de las instituciones, centros orientados únicamente a la atención de mujeres, se desconoce cuál sería el impacto en la satisfacción de esta clase de instituciones.

Es posible que el predominio de pacientes hombres $(n=179)$ sobre la cantidad de pacientes mujeres $(n=32)$, observado incluso en las instituciones mixtas (60 varones y $32 \mathrm{mu}-$ jeres) haga que los abordajes e instituciones estén más orientados al tratamiento de aquellos, sin tener en cuenta las especificidades de las mujeres tratadas. Estos hallazgos se vinculan con los reportados por Gómez Penedo y colaboradores (2015), quienes observaron un mayor nivel de satisfacción en pacientes varones con su tratamiento.

Estos resultados abren una serie de interrogantes que deberían ser explorados en posteriores estudios. ¿Son las instituciones abocadas únicamente al tratamiento de varones las más adecuadas para el abordaje de las adicciones y por ello reflejan una mayor satisfacción en sus pacientes?, ¿puede estar dicha adecuación basada en que haya un sesgo en los institutos mixtos debido a la marcada predominancia de varones, alejando a dichos tratamientos de las necesidades específicas de las mujeres?, ¿la creación de instituciones dirigidas exclusivamente al tratamiento de mujeres podría ser una alternativa que genere niveles de satisfacción mayores en la población femenina y potencialmente, mejores resultados?

E1 hecho de que el tratamiento haya sido o no interrupido en algún momento, no implicó diferencias en la satisfacción. Sin embargo en este punto hay que remarcar que una gran proporción de pacientes se encontraba en eta- 
pas tempranas del tratamiento. Como se describió en el apartado Participantes, el 25\% de los pacientes tenía menos de un mes de tratamiento, mientras que el 51\% tenía menos de 3 meses de tratamiento. A su vez, datos previos sugieren una alta tasa de abandono $(66.7 \%)$ de los tratamientos en estas poblaciones (OAD, 2009). Por ende, se podría hipotetizar que aquellas personas que interrumpieron el tratamiento y posteriormente lo retomaron, presentaban niveles de satisfacción por encima de aquellos que lo interrumpieron y luego lo abandonaron definitivamente.

Futuros estudios deberían indagar la relación entre niveles de satisfacción, interrupciones de los tratamientos y abandono, mediante estudios longitudinales, para establecer si la satisfacción se presenta como un predictor del abandono o mantenimiento del tratamiento.

Otras variables estudiadas tales como la ubicación geográfica de las instituciones y la presencia de características atípicas no mostraron diferencias relevantes con respecto a los niveles de satisfacción en los pacientes.

LIMITACIONES Y RESGUARDOS PARA LA INTERPRETACIÓN DE LOS RESULTADOS Y LAS CONCLUSIONES

Los resultados de esta investigación deben ser tomados con cautela por una serie de cuestiones. Por las características del muestreo utilizado (un muestreo aleatorio estratificado por conglomerados), el potencial principal de generalización de los resultados se centra en las instituciones que integran el sistema de atención que subsidia la SEDRONAR en el AMBA, población objeto de este estudio. Sin embargo, al focalizarse en esas instituciones, el estudio podría tener limitaciones en la generalización de los resultados a la población total, es decir, los pacientes tratados por abuso de sustancias en centros del AMBA (que incluye instituciones no subsidiadas por la SEDRONAR). Futuras investigaciones deberían explorar estas variables, utilizando muestreos aleatorios, a partir de la población total seleccionada, para aumentar su capacidad de generalización de los resultados.
Además, no se cuenta con información acerca de las propiedades psicométricas del instrumento utilizado. A pesar de que los análisis de confiabilidad, conducidos a partir de los datos de esta investigación arrojan resultados adecuados, serían necesarias a futuro investigaciones específicas con el fin de explorar la confiabilidad y validez del instrumento, para establecer sus propiedades psicométricas. Al no contar con estos datos, los resultados obtenidos con el instrumento deben ser interpretados con mayor cautela y en línea con el espíritu exploratorio de este estudio.

Si bien no apareció ningún indicador de que los sujetos se sintiesen coaccionados o inhibidos para expresar sus opiniones libremente, no se puede descartar que exista un efecto del contexto que podría llevarlos a puntuar los ítems en forma más positiva que su percepción real. Lo mismo puede suceder con la aquiescencia, tendencia a mostrar acuerdo con todo independientemente del contenido del ítem. Sin embargo, para evitar este fenómeno, algunos ítems (los pertenecientes al cluster Maltrato) fueron presentados en forma invertida sin mostrar una tendencia a una respuesta automatizada. En principio, este tipo de medidas tiende a reducir significativamente este efecto, sin embargo, no se puede descartar su presencia.

Por otra parte, los resultados de este estudio no se pueden cotejar con resultados anteriores, por lo cual no es posible establecer la potencial variación de los datos; para ello sería necesario repetirlo para poder comparar los datos a través del tiempo, conformando un estudio longitudinal.

Además, no se puede estimar con precisión la fiabilidad de la muestra, ya que se carece de datos sobre la fiabilidad en otra muestra equivalente, al no haberse encontrado otra investigación sobre satisfacción en adicciones a nivel nacional.

Finalmente, otra limitación es el alto grado de heterogeneidad de los tratamientos, que impidió la creación de una definición única de los mismos. Al haber diferentes propuestas terapéuticas, la satisfacción de los pacientes podría estar sesgada por las características de cada tratamiento específico. 
A futuro sería importante analizar en detalle las características específicas de los distintos tratamientos (marcos teóricos predominantes, intervenciones utilizadas, servicios terapéuticos ofrecidos) para poder crear sistemas de clasificación y definición más ex- haustivos en función de sus rasgos salientes. Solo después de ese paso se podría estudiar si dichas propuestas terapéuticas se asocian a diversos niveles de satisfacción y / o a mejores resultados.

GRÁFICO 1

FRECUENCIA DE SUJETOS SEGÚN TIEMPO DE TRATAMIENTO

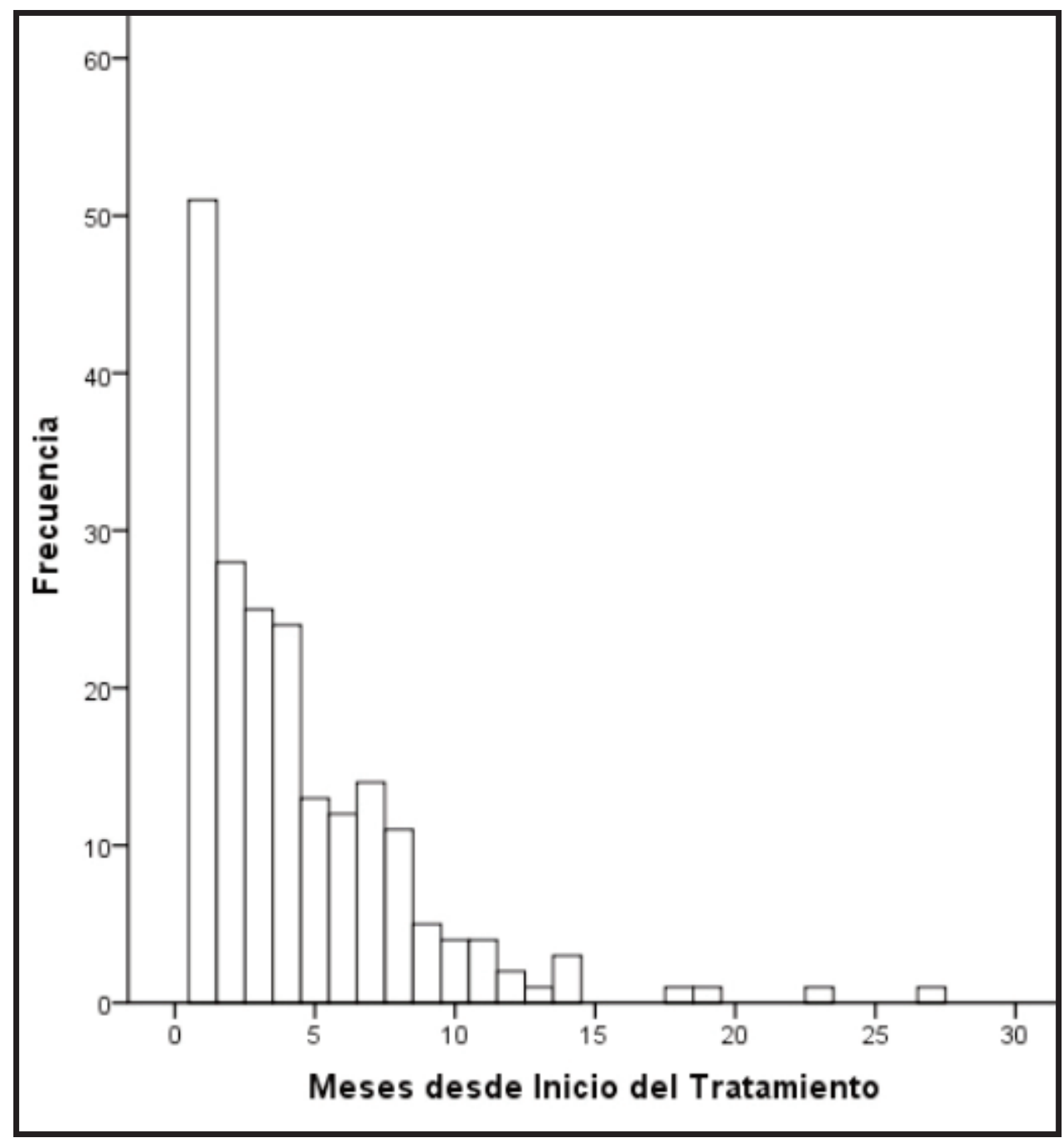




\section{TABLA 1}

CONSISTENCIA INTERNA Y HOMOGENIEDAD DE LOS ÍTEMS (CORRELACIÓN ÍTEM-TOTAL AJUSTADA) EN EL CUESTIONARIO TOTAL, CLUSTERS Y SUBCLUSTERS

\begin{tabular}{|c|c|c|}
\hline & $\alpha$ & $\begin{array}{l}\text { Correlación promedio } \\
\text { item-total ajustada }\end{array}$ \\
\hline Cuestionario total & .90 & .42 \\
\hline Cluster de Admisión e Ingreso & .60 & .44 \\
\hline Cluster de Ambiente General & .78 & .44 \\
\hline Subcluster de Ambiente Físico y Humano & .83 & .60 \\
\hline Subcluster de Maltrato & .86 & .69 \\
\hline Cluster de Comida & .87 & .77 \\
\hline Cluster de Evaluación del Personal & .71 & .38 \\
\hline Subcluster de Evaluación General del Personal & .78 & .59 \\
\hline Subcluster de Evaluación Específica del Personal & .62 & .33 \\
\hline Cluster de Tratamiento & .86 & .48 \\
\hline Subcluster de Características del Tratamiento & .76 & .45 \\
\hline Subcluster de Resultados del Tratamiento & .82 & .63 \\
\hline Subcluster de Dispositivos Complementarios & .66 & .44 \\
\hline
\end{tabular}


TABLA 2

MEDIAS DE SATISFACCIÓN EN LOS CLUSTERS ESTUDIADOS EN LA MUESTRA GENERAL Y SEGÚN CARACTERÍSTICAS DE LAS INSTITUCIONES

\begin{tabular}{|c|c|c|c|c|c|c|}
\hline & $A D$ & $A G$ & $\mathrm{CO}$ & EP & TR & EGT \\
\hline Muestra total $(N=216)$ & 4.18 & 4.31 & 3.74 & 4.23 & 4.17 & 4.38 \\
\hline$D E$ & 1.00 & 0.90 & 1.23 & 0.82 & 0.89 & 0.89 \\
\hline \multicolumn{7}{|l|}{ Tipo de institución } \\
\hline Tipo A $(n=9)$ & 3.89 & 4.02 & 2.56 & 3.86 & 3.73 & 4.00 \\
\hline Tipo $\mathrm{B}(n=80)$ & 4.21 & 4.28 & 3.77 & 4.25 & 4.10 & 4.29 \\
\hline Tipo C $(n=110)$ & 4.12 & 4.31 & 3.78 & 4.23 & 4.24 & 4.45 \\
\hline Tipo $\mathrm{D}(n=17)$ & 4.51 & 4.50 & 4.06 & 4.29 & 4.15 & 4.59 \\
\hline \multicolumn{7}{|l|}{ Ubicación } \\
\hline Sur de AMBA $(n=84)$ & 4.17 & 4.31 & 3.59 & 4.26 & 4.17 & 4.32 \\
\hline Norte de AMBA $(n=36)$ & 4.29 & 4.39 & 3.50 & 4.29 & 4.28 & 4.47 \\
\hline Oeste de AMBA $(n=40)$ & 4.03 & 4.16 & 4.17 & 4.20 & 4.12 & 4.41 \\
\hline $\mathrm{CABA}(n=56)$ & 4.21 & 4.32 & 3.82 & 4.16 & 4.11 & 4.41 \\
\hline \multicolumn{7}{|l|}{ Género de los pacientes $(n=216)$} \\
\hline Mixtas $(n=92)$ & 4.15 & 4.29 & 3.76 & 4.26 & 4.21 & 4.47 \\
\hline Solo varones $(n=124)$ & 4.21 & 4.32 & 3.71 & 4.18 & 4.10 & 4.27 \\
\hline \multicolumn{7}{|l|}{ Instituciones Atípicas } \\
\hline Religiosas $(n=15)$ & 4.16 & 4.19 & 3.04 & 4.26 & 4.24 & 4.47 \\
\hline Suspendidas $(n=10)$ & 3.90 & 4.25 & 3.30 & 4.10 & 4.19 & 4.10 \\
\hline \multicolumn{7}{|l|}{ Abordajes interrumpidos $(n=123)$} \\
\hline Interrumpidos $(n=29)$ & 4.11 & 4.28 & 3.78 & 4.16 & 4.01 & 4.46 \\
\hline No interrumpidos $(n=184)$ & 4.18 & 4.30 & 3.74 & 4.24 & 4.19 & 4.37 \\
\hline
\end{tabular}

\section{Notación}

AD: Admisión e Ingreso

AG: Ambiente General

CO: Comida

EP: Evaluación del Personal

TR: Tratamiento

EGT: Evaluación General del Tratamiento

Escala con valores de 1 (Muy insastifecho) a 5 (Muy satisfecho) 
TABLA 3

MEDIAS DE SATISFACCIÓN EN LOS SUBCLUSTERS ESTUDIADOS EN LA MUESTRA GENERAL Y SEGÚN CARACTERÍSTICAS DE LAS INSTITUCIONES

\begin{tabular}{|c|c|c|c|c|c|c|c|}
\hline & \multicolumn{2}{|c|}{$A G$} & \multicolumn{2}{|c|}{$\mathrm{EP}$} & \multicolumn{3}{|c|}{ TR } \\
\hline & (s)AFH & (s)Mal & (s)EGP & (s)EEP & (s)CT & $(\mathrm{s}) \mathrm{R}$ & (s)DC \\
\hline Muestra total & 4.00 & 4.73 & 4.32 & 4.17 & 4.13 & 4.42 & 3.92 \\
\hline$D E$ & .87 & .77 & .74 & .86 & .90 & .80 & .91 \\
\hline \multicolumn{8}{|l|}{ Tipo de institución } \\
\hline Tipo A $(n=9)$ & 3.46 & 4.70 & 3.94 & 3.83 & 3.58 & 4.20 & 3.29 \\
\hline Tipo $\mathrm{B}(n=80)$ & 4.02 & 4.66 & 4.35 & 4.20 & 4.04 & 4.40 & 3.88 \\
\hline Tipo $C(n=110)$ & 4.00 & 4.73 & 4.31 & 4.19 & 4.23 & 4.47 & 3.97 \\
\hline Tipo $\mathrm{D}(n=17)$ & 4.20 & 4.94 & 4.35 & 4.24 & 4.12 & 4.34 & 3.93 \\
\hline \multicolumn{8}{|l|}{ Ubicación } \\
\hline Sur de AMBA $(n=84)$ & 4.01 & 4.71 & 4.39 & 4.19 & 4.13 & 4.40 & 3.95 \\
\hline Norte de AMBA $(n=36)$ & 4.07 & 4.85 & 4.40 & 4.22 & 4.23 & 4.63 & 3.94 \\
\hline Oeste de AMBA $(n=40)$ & 3.93 & 4.51 & 4.17 & 4.25 & 4.09 & 4.37 & 3.89 \\
\hline $\mathrm{CABA}(n=56)$ & 3.99 & 4.79 & 4.26 & 4.11 & 4.07 & 4.36 & 3.86 \\
\hline \multicolumn{8}{|l|}{ Género de los pacientes $(n=216)$} \\
\hline Mixtas $(n=92)$ & 3.96 & 4.73 & 4.37 & 4.21 & 4.19 & 4.43 & 3.95 \\
\hline Solo varones $(n=124)$ & 4.07 & 4.71 & 4.25 & 4.15 & 4.03 & 4.41 & 3.86 \\
\hline \multicolumn{8}{|l|}{ Instituciones atípicas } \\
\hline Religiosas $(n=15)$ & 3.82 & 4.66 & 4.27 & 4.26 & 4.28 & 4.48 & 3.83 \\
\hline Suspendidas $(n=10)$ & 3.79 & 4.84 & 4.22 & 4.02 & 3.99 & 4.62 & 4.18 \\
\hline \multicolumn{8}{|l|}{ Abordajes interrumpidos $(n=123)$} \\
\hline Interrumpidos $(n=29)$ & 4.00 & 4.70 & 4.27 & 4.10 & 3.95 & 4.27 & 3.79 \\
\hline No interrumpidos $(n=184)$ & 4.00 & 4.72 & 4.32 & 4.20 & 4.15 & 4.45 & 3.93 \\
\hline
\end{tabular}

Notación:

(s)AFH: Ambiente Físico y Humano

(s)Mal: Maltrato

(s)EGP: Evaluación General del Personal

(s)EEP: Evaluación Específica del Personal

(s)CT: Características del Tratamiento

(s)R: Resultados

(s)DC: Dispositivos Complementarios

AG: Ambiente General

EP: Evaluación del Personal

TR: Tratamiento 


\section{REFERENCIAS BIBLIOGRÁFICAS}

Ankuta, G.Y. \& Abeles, N. (1993). Client satisfaction, clinical significance and meaningful change in psychotherapy. Professional Psychology: Research and Practice, 24(1), 70-74. http: //dx.doi.org/10.1037/0735-7028.24.1.70

Arenas, Y., Fuentes, V. \& Campos, C. (1993). Grado de satisfacción usuaria de la consulta EPASRN del Servicio de Pediatría del CEDIUC [Degree of satisfaction of the users of the treatments EPAS-RN in the pediatric service of CEDIUC]. EPAS, 10(1), 15-17.

Bunge, E. (2012). Satisfacción de los usuarios con los servicios de salud mental para niños y adolescentes desde la perspectiva del paciente y el cuidador [Satisfaction of the users of mental health services for children and teenagers from clients and caregivers perspective]. Tesis doctoral no publicada. Universidad de Palermo. Ciudad Autónoma de Buenos Aires, Argentina.

Cernovsky, Z.Z., O’Reilly, R.L. \& Pennington, M. (1997). Sensation seeking scales and consumer satisfaction with a substance abuse treatment program. Journal of Clinical Psychology, 53(8), 779-784. http://dx.doi.org/10.1002/(SICI)109 7-4679(199711)53:7\%3C779::AID-JCLP16\% 3E3.0.CO;2-I

Consejo Nacional para el Control de Estupefacientes (s.f.). Experiencia chilena en evaluación de programas de tratamiento y rehabilitación del consumo problemático de sustancias psicoactivas [Chilean experience in evaluation of treatment programs and rehabilitation of the problematic use of psychoactive substances]. Santiago de Chile: Editorial Atenas.

Donovan, D.M., Kadden, R M., Diclemente, C.C. \& Carroll, K.M. (2002). Client satisfaction with three therapies in the treatment of alcohol dependence: Results from Project MATCH. The American Journal on Addictions, 11, 291307. http://dx.doi.org/10.1080/105504902900 88090

Frick, K.M., Loessl, B., Brueck, R.K., Kriston, L., Jaehne, A., Riemann, D. et al. (2011). What works for patients in outpatient treatment for alcohol addiction? An explorative study into clients' evaluation of subjective factors and therapy satisfaction. Substance abuse: Research and Treatment, 5, 27-34.

Gómez Penedo, J.M., Barreiro, D. \& Roussos, A.J. (2015). Relación de las características de pacientes con trastornos por abuso de sustancias con sus niveles de satisfacción por el tratamiento [Relationships among baseline characteristics in patients with drug abuse disordes and there levels of satisfaction with treatment]. Vertex, Revista Argentina de Psiquiatría, 26, 92-99.

Holcomb, W.R., Parker, J.C., Leong, G.B., Thiele, J. \& Higdon, J. (1998). Customer satisfaction and self-reported treatment outcomes among psychiatric inpatients. Psychiatric Services, 49 (7), 929-934. http://dx.doi.org/10.1176/ps.49. 7.929

Kelly, S.M., O’Grady, K.E., Brown, B.S., Mitchell, S.G. \& Schwartz, R.P. (2010). The role of patient satisfaction in methadone treatment. The American Journal of Drug and Alcohol Abuse, 36, 150-154. http://dx.doi.org/10.3109/00952 991003736371

Lambert, M.J. \& Ogles, B.M. (2004). The efficacy and effectiveness of psychotherapy. En M.J. Lambert (Comp.), Bergin \& Garfield's handbook of psychotherapy and behavior change (5ta. ed., pp. 139-193). New York: Wiley.

Marchand, K.I., Oviedo-Joekes, E., Guh, D., Brissette, S., Marsh, D.C. \& Schechter, M.T. (2011). Client satisfaction among participants in a randomized trial comparing oral methadone and injectable diacetylmorphine for long-term opioid-dependency. BMC Health Services Research, 11, 174. http://dx.doi.org/10.1186/147 2-6963-11-174

McLellan, A.T. \& Hunkeler, E. (1998). Patient satisfaction and outcomes in alcohol and drug abuse treatment. Psychiatric Services, 49(5), 573-575.

Morris, Z.S. \& Gannon, M. (2008). Drug misuse treatment services in Scotland: Predicting outcomes. International Journal for Quality in Health Care, 20(4), 271-276. http://dx.doi.org/ 10.1093/intqhe/mzn019 
New York State Office of Alcoholism and Substance Abuse Service Bureau of Addiction Treatment Centers (s.f.). Patient Satisfaction Survey. Recuperado el 10 de septiembre de 2012 de http://www.oasas.ny.gov/GSI/QI/documents/ATCSurvey.pdf

Observatorio Argentino de Drogas - SEDRONAR (2009). Evaluación de tratamientos en pacientes por abuso de sustancias [Treatments evaluation in patients with substance abuse]. Ciudad Autónoma de Buenos Aires, Argentina.

Oficina de las Naciones Unidas contra la Droga y el Delito (2003). Manual sobre el tratamiento del abuso de drogas [Treatment manual for drug abuse].Viena: Autor.

Oficina de las Naciones Unidas contra la Droga y el Delito (2012). Informe mundial sobre las drogas 2012 [World Drug Report 2012]. Recuperado el 12 de marzo de 2013 de http://www. unodc.org/documents/data-and-analysis/WDR 2012/Executive_summary_spanish.pdf

Organización Mundial de la Salud (2003). Mejora de la calidad de la salud mental. Conjunto de guías sobre servicios y políticas de salud mental [Improving the quality of mental health. Set of guidelines on services and mental health policies]. Ginebra: Autor.
Organización Panamericana de la Salud (2009). Epidemiología del uso de drogas en América Latina y el Caribe: Un enfoque de salud públi$c a$ [Epidemiology of drug use in Latin America and the Caribbean: A public health approach]. Washington, DC: Autor.

Pekarik, G. \& Wolff, C.B. (1996). Relationship of satisfaction to symptom change, follow-up adjustment, and clinical significance. Professional Psychology: Research and Practice, 27(2), 202-208. http://dx.doi.org/10.1037/0735-7028. 27.2.202

Rojas Valero, M.J. \& Espinoza Paul, L.S. (2008). Consejo psicológico y psicoterapia motivacional en drogodependencias: Evaluación de las intervenciones del programa "Lugar de Escucha"- CEDRO [Psychological counseling and motivational psychotherapy in drug addictions: Evaluation of the program interventions "Listening Place" - CEDRO]. Adicciones, 20(2), 185-196.

Schulte, S.J., Meier, P.S. \& Stirling, J. (2011). Dual diagnosis clients' treatment satisfaction - a systematic review. BMC Psychiatry, 11(64), 1-12.

Zhang, Z., Gerstein, D.R. \& Friedmann, P.D. (2008). Patient satisfaction and sustained outcomes of drug abuse treatment. Journal of Health Psychology, 13(3), 388-400.

Facultad de Psicología Universidad de Buenos Aires (UBA) Consejo Nacional de Investigaciones Científicas y Técnicas (CONICET) Buenos Aires - República Argentina

Fecha de recepción: 29 de abril de 2016 Fecha de aceptación: 12 de septiembre de 2016 